\title{
Generalized retarded nonlinear integral inequalities involving iterated integrals and an application
}

\author{
Wu-Sheng Wang ${ }^{1 *}$, Deqing Huang ${ }^{2}$ and Xuefang $\mathrm{Li}^{2}$
}

\section{"Correspondence:} wang4896@126.com

'Department of Mathematics, Hechi University, Yizhou, Guangxi 546300, P.R. China

Full list of author information is available at the end of the article

\begin{abstract}
In this work, some new generalized retarded nonlinear integral inequalities, which include nonlinear composite functions of unknown functions between iterated integrals, are discussed. By adopting novel analysis techniques, the upper bounds of the embedded unknown functions are estimated explicitly. The derived results can be applied in the study of differential-integral equations and some practical problems in engineering.
\end{abstract}

MSC: 26D15; 26D20; 34A12

Keywords: integral inequality; iterated integrals; analysis technique; estimation

\section{Introduction}

Integral inequality that provides an explicit bound to the unknown function furnishes a handy tool to investigate qualitative properties of solutions of differential and integral equations. One of the best known and widely used inequalities in the study of nonlinear differential equations is Gronwall-Bellman inequality $[1,2]$, which can be stated as follows: If $u$ and $f$ are nonnegative continuous functions on an interval $[a, b]$ satisfying

$$
u(t) \leq c+\int_{a}^{t} f(s) u(s) d s, \quad t \in[a, b],
$$

for some constant $c \geq 0$, then

$$
u(t) \leq c \exp \left(\int_{a}^{t} f(s) d s\right), \quad t \in[a, b] .
$$

It has become one of the very few classical and most influential results in the theory and applications of inequalities. Because of its fundamental importance, over the years, many generalizations and analogous results of (1.1) have been established, such as [3-23].

Among these references, Bainov et al. [7, p.107] considered the following interesting Gronwall-type inequality

$$
\begin{aligned}
u(t) \leq & a(t)+\sum_{i=1}^{n} \int_{\alpha}^{t} f_{1}\left(t, t_{1}\right)\left(\int_{\alpha}^{t_{1}} f_{2}\left(t_{1}, t_{2}\right) \cdots\right. \\
& \left.\times\left(\int_{\alpha}^{t_{i-1}} f_{i}\left(t_{i-1}, t_{i}\right) u\left(t_{i}\right) d t_{i}\right) \cdots\right) d t_{1},
\end{aligned}
$$

\section{Springer}

(0) 2013 Wang et al.; licensee Springer. This is an Open Access article distributed under the terms of the Creative Commons Attribution License (http://creativecommons.org/licenses/by/2.0), which permits unrestricted use, distribution, and reproduction in any medium, provided the original work is properly cited. 
in which the unknown function only exists in the innermost layer of iterated integrals. In $2005 \mathrm{Kim}$ [8] considered analogous Gronwall-type integral inequalities involving iterated integrals by replacing the unknown function $u$ in the right-hand side of (1.2) with $u^{p}$ for some constant $p$. In 2007, Agarwal et al. [10] investigated some nonlinear retarded inequalities with iterated integrals to extend Kim's results in [8],

$$
\begin{aligned}
\varphi(u(t)) \leq & a(t)+\int_{\phi(\alpha)}^{\phi(t)} p_{1}\left(t_{1}\right) f_{1}\left(t_{1}\right) u\left(t_{1}\right) g\left(u\left(t_{1}\right)\right) d t_{1} \\
& +\sum_{i=2}^{n} \int_{\phi(\alpha)}^{\phi(t)} p_{1}\left(t_{1}\right)\left(\int _ { \phi ( \alpha ) } ^ { \phi ( t _ { 1 } ) } p _ { 2 } ( t _ { 2 } ) \left(\cdots \left(\int_{\phi(\alpha)}^{\phi\left(t_{i-2}\right)} p_{i-1}\left(t_{i-1}\right)\right.\right.\right. \\
& \left.\left.\left.\times\left(\int_{\phi(\alpha)}^{\phi\left(t_{i-1}\right)} p_{i}\left(t_{i}\right) f_{i}\left(t_{i}\right) u\left(t_{i}\right) g\left(u\left(t_{i}\right)\right) d t_{i}\right) d t_{i-1}\right) \cdots\right) d t_{2}\right) d t_{1},
\end{aligned}
$$

which include the composite functions of unknown function only in the innermost layer of iterated integrals.

In 2011, Abdeldaim et al. [11] studied some new integral inequalities of GronwallBellman-Pachpatte-type such as

$$
u(t) \leq u_{0}+\int_{0}^{t} f(s) u(s)\left[u(s)+\int_{0}^{s} h(\tau)\left[u(\tau)+\int_{0}^{\tau} g(\xi) u(\xi) d \xi\right] d \tau\right] d s,
$$

and

$$
u(t) \leq u_{0}+\int_{0}^{t}[f(s) u(s)+q(s)] d s+\int_{0}^{t} f(s) u(s)\left[u(s)+\int_{0}^{s} g(\tau) u(\tau) d \tau\right] d s,
$$

which include the composite functions of unknown functions in every layer of iterated integrals, but the iterated integrals are double integrals.

In this paper, we extend certain results that were proved in [7-11] to obtain new generalizations of formerly famous Gronwall-Bellman-Pachpatte-type inequalities. There are not only composite functions of unknown functions in iterated integrals on the right hand side of our inequalities, but also the composite functions of unknown function exist in every layer of the iterated integrals. In this work, we give the upper bounds of the embedded unknown functions by adopting novel analysis techniques in three different scenarios and illustrate an application of our results, which verifies that our results are handy tools to study the qualitative properties of nonlinear differential equations and integral equations.

\section{Main result}

In this section, we state and prove some new integral inequalities of Gronwall-BellmanPachpatte-type, which can be used in the analysis of various problems in the theory of nonlinear ordinary differential and integral equations.

First, we give five assumptions for functions that will appear in our main results.

1. $u(t)$ and $a(t)$ are nonnegative and continuous functions on $\left[t_{0},+\infty\right)$. In addition, $a(t)$ is nondecreasing;

2. $f_{i}(t, s), i=1,2,3$ are nonnegative and continuous functions for $t_{0} \leq s \leq t \leq+\infty$, and nondecreasing in $t$ for fixed $s \in\left[t_{0},+\infty\right)$;

3. $w(u)$ is a nondecreasing and continuous function on $[0,+\infty)$ with $w(u)>0$ for $u>0$; 
4. $\varphi(u)$ is an increasing continuous function with $\varphi(u)>0$ for $u>0$ and $\varphi(\infty)=\infty$;

5. $\alpha(t)$ is a continuous, differentiable and nondecreasing function on $\left[t_{0},+\infty\right)$ with $\alpha(t)<t, \alpha\left(t_{0}\right)=t_{0}$.

In order to clearly present our main idea, we first consider a class of simple integral inequalities, namely, the composite function of unknown function $\varphi(u)$ is involved in the innermost layer of iterated integrals only.

Theorem 1 Assume that Assumptions 1-5 and the following inequality hold

$$
\begin{aligned}
\varphi(u(t)) \leq & a(t)+\int_{\alpha\left(t_{0}\right)}^{\alpha(t)} f_{1}(t, s) w(u(s)) d s \\
& +\int_{\alpha\left(t_{0}\right)}^{\alpha(t)} f_{1}(t, s)\left(\int_{t_{0}}^{s} f_{2}(s, \tau) w(u(\tau)) d \tau\right) d s \\
& +\int_{\alpha\left(t_{0}\right)}^{\alpha(t)} f_{1}(t, s)\left(\int_{t_{0}}^{s} f_{2}(s, \tau)\left(\int_{t_{0}}^{\tau} f_{3}(\tau, \xi) w(u(\xi)) d \xi\right) d \tau\right) d s
\end{aligned}
$$

for $t \in\left[t_{0}, \infty\right)$. Then we have

$$
u(t) \leq \varphi^{-1}\left(W^{-1}\left(U_{1}(t)\right)\right), \quad \forall t \in\left[t_{0}, T_{1}\right)
$$

where

$$
\begin{aligned}
U_{1}(t):= & W(a(t))+\int_{\alpha\left(t_{0}\right)}^{\alpha(t)} f_{1}(t, s) d s+\int_{\alpha\left(t_{0}\right)}^{\alpha(t)} f_{1}(t, s)\left(\int_{t_{0}}^{s} f_{2}(s, \tau) d \tau\right) d s \\
& +\int_{\alpha\left(t_{0}\right)}^{\alpha(t)} f_{1}(t, s)\left(\int_{t_{0}}^{s} f_{2}(s, \tau)\left(\int_{t_{0}}^{\tau} f_{3}(\tau, \xi) d \xi\right) d \tau\right) d s, \\
W(u):= & \int_{u_{0}}^{u} \frac{d s}{w\left(\varphi^{-1}(s)\right)}, \quad u>u_{0},
\end{aligned}
$$

and $\varphi^{-1}, W^{-1}$ are the inverse functions of $\varphi, W$, respectively, and

$$
T_{1}:=\max \left\{t \in\left[t_{0},+\infty\right) \mid U_{1}(t) \in \operatorname{Dom}\left(W^{-1}\right)\right\}
$$

Proof Choose $T \in\left[t_{0}, T_{1}\right)$ arbitrarily. For $\forall t \in\left[t_{0}, T\right]$, we obtain that

$$
\begin{aligned}
\varphi(u(t)) \leq & a(T)+\int_{\alpha\left(t_{0}\right)}^{\alpha(t)} f_{1}(T, s) w(u(s)) d s \\
& +\int_{\alpha\left(t_{0}\right)}^{\alpha(t)} f_{1}(T, s)\left(\int_{t_{0}}^{s} f_{2}(s, \tau) w(u(\tau)) d \tau\right) d s \\
& +\int_{\alpha\left(t_{0}\right)}^{\alpha(t)} f_{1}(T, s)\left(\int_{t_{0}}^{s} f_{2}(s, \tau)\left(\int_{t_{0}}^{\tau} f_{3}(\tau, \xi) w(u(\xi)) d \xi\right) d \tau\right) d s,
\end{aligned}
$$

from (2.1), by Assumption 2 that $f_{i}(t, s)$ are nondecreasing in $t$. Let $z_{1}(t)$ be the right-hand side of (2.5), which is a positive and nondecreasing function on $\left[t_{0}, T\right]$ with $z_{1}\left(t_{0}\right)=a(T)$. Then (2.5) can be written as

$$
u(t) \leq \varphi^{-1}\left(z_{1}(t)\right), \quad \forall t \in\left[t_{0}, T\right]
$$


since the inverse function $\varphi^{-1}$ of $\varphi$ exists by Assumption 4. From (2.5) and (2.6), we can obtain that

$$
\begin{aligned}
\frac{d z_{1}(t)}{d t} \leq & \alpha^{\prime}(t) f_{1}(T, \alpha(t)) w\left(\varphi^{-1}\left(z_{1}(t)\right)\right) \\
& +\alpha^{\prime}(t) f_{1}(T, \alpha(t)) \int_{t_{0}}^{\alpha(t)} f_{2}(\alpha(t), \tau) w\left(\varphi^{-1}\left(z_{1}(\tau)\right)\right) d \tau \\
& +\alpha^{\prime}(t) f_{1}(T, \alpha(t)) \int_{t_{0}}^{\alpha(t)} f_{2}(\alpha(t), \tau)\left(\int_{t_{0}}^{\tau} f_{3}(\tau, \xi) w\left(\varphi^{-1}\left(z_{1}(\xi)\right)\right) d \xi\right) d \tau
\end{aligned}
$$

for all $t \in\left[t_{0}, T\right]$. Applying the monotonicity of $w, \varphi$ and $z_{1},(2.7)$ can be written as

$$
\begin{aligned}
\frac{d z_{1}(t)}{w\left(\varphi^{-1}\left(z_{1}(t)\right)\right)} \leq & \left(\alpha^{\prime}(t) f_{1}(T, \alpha(t))+\alpha^{\prime}(t) f_{1}(T, \alpha(t)) \int_{t_{0}}^{\alpha(t)} f_{2}(\alpha(t), \tau) d \tau\right. \\
& \left.+\alpha^{\prime}(t) f_{1}(T, \alpha(t)) \int_{t_{0}}^{\alpha(t)} f_{2}(\alpha(t), \tau)\left(\int_{t_{0}}^{\tau} f_{3}(\tau, \xi) d \xi\right) d \tau\right) d t
\end{aligned}
$$

for all $t \in\left[t_{0}, T\right]$. Integrating both sides of the above inequality from $t_{0}$ to $t$, we can obtain that

$$
\begin{aligned}
W\left(z_{1}(t)\right) \leq & W\left(z_{1}\left(t_{0}\right)\right)+\int_{\alpha\left(t_{0}\right)}^{\alpha(t)} f_{1}(T, s) d s \\
& +\int_{\alpha\left(t_{0}\right)}^{\alpha(t)} f_{1}(T, s)\left(\int_{t_{0}}^{s} f_{2}(s, \tau) d \tau\right) d s \\
& +\int_{\alpha\left(t_{0}\right)}^{\alpha(t)} f_{1}(T, s)\left(\int_{t_{0}}^{s} f_{2}(s, \tau)\left(\int_{t_{0}}^{\tau} f_{3}(\tau, \xi) d \xi\right) d \tau\right) d s, \quad \forall t \in\left[t_{0}, T\right],
\end{aligned}
$$

where $W$ is defined as (2.4). In consequence, we get that

$$
\begin{aligned}
u(t) \leq & \varphi^{-1}\left(W^{-1}(W(a(T))\right. \\
& +\int_{\alpha\left(t_{0}\right)}^{\alpha(t)} f_{1}(T, s) d s+\int_{\alpha\left(t_{0}\right)}^{\alpha(t)} f_{1}(T, s)\left(\int_{t_{0}}^{s} f_{2}(s, \tau) d \tau\right) d s \\
& \left.\left.+\int_{\alpha\left(t_{0}\right)}^{\alpha(t)} f_{1}(T, s)\left(\int_{t_{0}}^{s} f_{2}(s, \tau)\left(\int_{t_{0}}^{\tau} f_{3}(\tau, \xi) d \xi\right) d \tau\right) d s\right)\right), \quad \forall t \in\left[t_{0}, T\right],
\end{aligned}
$$

by (2.6) and (2.9). Let $t=T$ on both hand sides of (2.10), then we have that

$$
\begin{aligned}
u(T) \leq & \varphi^{-1}\left(W ^ { - 1 } \left(W(a(T))+\int_{\alpha\left(t_{0}\right)}^{\alpha(T)} f_{1}(T, s) d s\right.\right. \\
& +\int_{\alpha\left(t_{0}\right)}^{\alpha(T)} f_{1}(T, s)\left(\int_{t_{0}}^{s} f_{2}(s, \tau) d \tau\right) d s \\
& \left.\left.+\int_{\alpha\left(t_{0}\right)}^{\alpha(T)} f_{1}(T, s)\left(\int_{t_{0}}^{s} f_{2}(s, \tau)\left(\int_{t_{0}}^{\tau} f_{3}(\tau, \xi) d \xi\right) d \tau\right) d s\right)\right) .
\end{aligned}
$$


Thus, we obtain that

$$
u(t) \leq \varphi^{-1}\left(W^{-1}\left(U_{1}(t)\right)\right)
$$

from (2.11), where $U_{1}(t)$ is defined as (2.3), since $T$ is chosen arbitrarily.

Next, consider a more general scenario: the composite function of unknown function exists not only in the innermost layer of iterated integrals, but also in the outermost layer of iterated integrals.

Theorem 2 Assume that Assumptions 1-5 and the following inequality hold

$$
\begin{aligned}
\varphi(u(t)) \leq & a(t)+\int_{\alpha\left(t_{0}\right)}^{\alpha(t)} f_{1}(t, s) w(u(s)) d s \\
& +\int_{\alpha\left(t_{0}\right)}^{\alpha(t)} f_{1}(t, s) w(u(s))\left(\int_{t_{0}}^{s} f_{2}(s, \tau) w(u(\tau)) d \tau\right) d s \\
& +\int_{\alpha\left(t_{0}\right)}^{\alpha(t)} f_{1}(t, s) w(u(s))\left(\int_{t_{0}}^{s} f_{2}(s, \tau)\left(\int_{t_{0}}^{\tau} f_{3}(\tau, \xi) w(u(\xi)) d \xi\right) d \tau\right) d s .
\end{aligned}
$$

Then the integral inequality (2.12) implies that

$$
u(t) \leq \varphi^{-1}\left\{W^{-1}\left[J^{-1}\left(U_{2}(t)\right)\right]\right\}, \quad \forall t \in\left[t_{0}, T_{2}\right],
$$

where $W$ is defined in (2.4) and

$$
\begin{aligned}
J(u):= & \int_{u_{0}}^{u} \frac{d s}{w\left(\varphi^{-1}\left(W^{-1}(s)\right)\right)}, \quad u>u_{0}, \\
U_{2}(t):= & J\left(W(a(t))+\int_{\alpha\left(t_{0}\right)}^{\alpha(t)} f_{1}(t, s) d s\right)+\int_{\alpha\left(t_{0}\right)}^{\alpha(t)} f_{1}(t, s)\left(\int_{t_{0}}^{s} f_{2}(s, \tau) d \tau\right) d s \\
& +\int_{\alpha\left(t_{0}\right)}^{\alpha(t)} f_{1}(t, s)\left(\int_{t_{0}}^{s} f_{2}(s, \tau)\left(\int_{t_{0}}^{\tau} f_{3}(\tau, \xi) d \xi\right) d \tau\right) d s,
\end{aligned}
$$

and $\varphi^{-1}, W^{-1}, J^{-1}$ are the inverse functions of $\varphi, W, J$, respectively, and

$$
T_{2}:=\max \left\{t \in\left[t_{0},+\infty\right) \mid U_{2}(t) \in \operatorname{Dom}\left(J^{-1}\right), J^{-1}\left(U_{2}(t)\right) \in \operatorname{Dom}\left(W^{-1}\right)\right\}
$$

Proof Choose $T \in\left[t_{0}, T_{2}\right]$ arbitrarily. For $\forall t \in\left[t_{0}, T\right]$, from (2.12), we have that

$$
\begin{aligned}
\varphi(u(t)) \leq & a(T)+\int_{\alpha\left(t_{0}\right)}^{\alpha(t)} f_{1}(T, s) w(u(s)) d s \\
& +\int_{\alpha\left(t_{0}\right)}^{\alpha(t)} f_{1}(T, s) w(u(s))\left(\int_{t_{0}}^{s} f_{2}(s, \tau) w(u(\tau)) d \tau\right) d s \\
& +\int_{\alpha\left(t_{0}\right)}^{\alpha(t)} f_{1}(T, s) w(u(s))\left(\int_{t_{0}}^{s} f_{2}(s, \tau)\left(\int_{t_{0}}^{\tau} f_{3}(\tau, \xi) w(u(\xi)) d \xi\right) d \tau\right) d s
\end{aligned}
$$


by Assumption 2. Denote the right-hand side of (2.16) by $z_{2}(t)$, which can be proved that it is positive and nondecreasing on $\left[t_{0}, T\right]$ with $z_{2}\left(t_{0}\right)=a(T)$. Then (2.16) can be written as

$$
u(t) \leq \varphi^{-1}\left(z_{2}(t)\right), \quad \forall t \in\left[t_{0}, T\right]
$$

by Assumption 4. From (2.16) and (2.17), we obtain that

$$
\begin{aligned}
\frac{d z_{2}(t)}{d t} \leq & \alpha^{\prime}(t) f_{1}(T, \alpha(t)) w\left(\varphi^{-1}\left(z_{2}(t)\right)\right)+\alpha^{\prime}(t) f_{1}(T, \alpha(t)) \\
& \times w\left(\varphi^{-1}\left(z_{2}(t)\right)\right) \int_{t_{0}}^{\alpha(t)} f_{2}(\alpha(t), \tau) w\left(\varphi^{-1}\left(z_{2}(\tau)\right)\right) d \tau+\alpha^{\prime}(t) f_{1}(T, \alpha(t)) \\
& \times w\left(\varphi^{-1}\left(z_{2}(t)\right)\right) \int_{t_{0}}^{\alpha(t)} f_{2}(\alpha(t), \tau)\left(\int_{t_{0}}^{\tau} f_{3}(\tau, \xi) w\left(\varphi^{-1}\left(z_{2}(\xi)\right)\right) d \xi\right) d \tau,
\end{aligned}
$$

By the property of monotonicity of functions $w, \varphi$ and $z_{2}$, we can obtain that

$$
\begin{aligned}
\frac{d z_{2}(t)}{w\left(\varphi^{-1}\left(z_{2}(t)\right)\right)} \leq & \left(\alpha^{\prime}(t) f_{1}(T, \alpha(t))\right. \\
& +\alpha^{\prime}(t) f_{1}(T, \alpha(t)) \int_{t_{0}}^{\alpha(t)} f_{2}(\alpha(t), \tau) w\left(\varphi^{-1}\left(z_{2}(\tau)\right)\right) d \tau \\
& +\alpha^{\prime}(t) f_{1}(T, \alpha(t)) \int_{t_{0}}^{\alpha(t)} f_{2}(\alpha(t), \tau) \\
& \left.\times\left(\int_{t_{0}}^{\tau} f_{3}(\tau, \xi) w\left(\varphi^{-1}\left(z_{2}(\xi)\right)\right) d \xi\right) d \tau\right) d t
\end{aligned}
$$

from (2.18). Integrating both sides of the above inequality from $t_{0}$ to $t$, we have that

$$
\begin{aligned}
W\left(z_{2}(t)\right) \leq & W\left(z_{2}\left(t_{0}\right)\right)+\int_{\alpha\left(t_{0}\right)}^{\alpha(t)} f_{1}(T, s) d s \\
& +\int_{\alpha\left(t_{0}\right)}^{\alpha(t)} f_{1}(T, s)\left(\int_{t_{0}}^{s} f_{2}(s, \tau) w\left(\varphi^{-1}\left(z_{2}(\tau)\right)\right) d \tau\right) d s \\
& +\int_{\alpha\left(t_{0}\right)}^{\alpha(t)} f_{1}(T, s)\left(\int_{t_{0}}^{s} f_{2}(s, \tau)\left(\int_{t_{0}}^{\tau} f_{3}(\tau, \xi) w\left(\varphi^{-1}\left(z_{2}(\xi)\right)\right) d \xi\right) d \tau\right) d s \\
\leq & W\left(z_{2}\left(t_{0}\right)\right)+\int_{\alpha\left(t_{0}\right)}^{\alpha(T)} f_{1}(T, s) d s \\
& +\int_{\alpha\left(t_{0}\right)}^{\alpha(t)} f_{1}(T, s)\left(\int_{t_{0}}^{s} f_{2}(s, \tau) w\left(\varphi^{-1}\left(z_{2}(\tau)\right)\right) d \tau\right) d s \\
& +\int_{\alpha\left(t_{0}\right)}^{\alpha(t)} f_{1}(T, s)\left(\int_{t_{0}}^{s} f_{2}(s, \tau)\left(\int_{t_{0}}^{\tau} f_{3}(\tau, \xi) w\left(\varphi^{-1}\left(z_{2}(\xi)\right)\right) d \xi\right) d \tau\right) d s
\end{aligned}
$$

for all $t \in\left[t_{0}, T\right]$, where $W$ is given in (2.4). Let $v_{1}(t)$ denote the right-hand side of (2.19), which can be proved to be a positive and nondecreasing function on $\left[t_{0}, T\right]$ with $v_{1}\left(t_{0}\right)=$ $W(a(T))+\int_{\alpha\left(t_{0}\right)}^{\alpha(T)} f_{1}(T, s) d s$. Then (2.19) is equivalent to

$$
z_{2}(t) \leq W^{-1}\left(v_{1}(t)\right), \quad \forall t \in\left[t_{0}, T\right]
$$


Differentiating $v_{1}$, we get that

$$
\begin{aligned}
\frac{d \nu_{1}(t)}{d t} \leq & \alpha^{\prime}(t) f_{1}(T, \alpha(t)) \int_{t_{0}}^{\alpha(t)} f_{2}(\alpha(t), \tau) w\left(\varphi^{-1}\left(W^{-1}\left(v_{1}(\tau)\right)\right)\right) d \tau \\
& +\alpha^{\prime}(t) f_{1}(T, \alpha(t)) \int_{t_{0}}^{\alpha(t)} f_{2}(\alpha(t), \tau) \\
& \times\left(\int_{t_{0}}^{\tau} f_{3}(\tau, \xi) w\left(\varphi^{-1}\left(W^{-1}\left(v_{1}(\xi)\right)\right)\right) d \xi\right) d \tau
\end{aligned}
$$

using (2.20), for all $t \in\left[t_{0}, T\right]$. By (2.21) and the monotonicity of $w, \varphi^{-1}, W^{-1}$ and $v_{1}$, we further obtain that

$$
\begin{aligned}
\frac{d \nu_{1}(t)}{w\left(\varphi^{-1}\left(W^{-1}\left(v_{1}(t)\right)\right)\right)} \leq & \left(\alpha^{\prime}(t) f_{1}(T, \alpha(t)) \int_{t_{0}}^{\alpha(t)} f_{2}(\alpha(t), \tau) d \tau\right. \\
& \left.+\alpha^{\prime}(t) f_{1}(T, \alpha(t)) \int_{t_{0}}^{\alpha(t)} f_{2}(\alpha(t), \tau)\left(\int_{t_{0}}^{\tau} f_{3}(\tau, \xi) d \xi\right) d \tau\right) d t
\end{aligned}
$$

for all $t \in\left[t_{0}, T\right]$. Integrating both sides of the above inequality from $t_{0}$ to $t$, we obtain

$$
\begin{aligned}
J\left(v_{1}(t)\right) \leq & J\left(v_{1}\left(t_{0}\right)\right)+\int_{\alpha\left(t_{0}\right)}^{\alpha(t)} f_{1}(T, s)\left(\int_{t_{0}}^{s} f_{2}(s, \tau) d \tau\right) d s \\
& +\int_{\alpha\left(t_{0}\right)}^{\alpha(t)} f_{1}(T, s)\left(\int_{t_{0}}^{s} f_{2}(s, \tau)\left(\int_{t_{0}}^{\tau} f_{3}(\tau, \xi) d \xi\right) d \tau\right) d s,
\end{aligned}
$$

for all $t \in\left[t_{0}, T\right]$, where $J$ is defined by (2.14). Hence, inequalities (2.17), (2.20) and (2.22) yield that

$$
\begin{aligned}
u(t) \leq & \varphi^{-1}\left\{z_{2}(t)\right\} \leq \varphi^{-1}\left\{W^{-1}\left[v_{1}(t)\right]\right\} \\
= & \varphi^{-1}\left\{W ^ { - 1 } \left[J^{-1}(J(W(a(T))\right.\right. \\
& \left.+\int_{\alpha\left(t_{0}\right)}^{\alpha(T)} f_{1}(T, s) d s\right)+\int_{\alpha\left(t_{0}\right)}^{\alpha(t)} f_{1}(T, s)\left(\int_{t_{0}}^{s} f_{2}(s, \tau) d \tau\right) d s \\
& \left.\left.\left.+\int_{\alpha\left(t_{0}\right)}^{\alpha(t)} f_{1}(T, s)\left(\int_{t_{0}}^{s} f_{2}(s, \tau)\left(\int_{t_{0}}^{\tau} f_{3}(\tau, \xi) d \xi\right) d \tau\right) d s\right)\right]\right\}, \quad \forall t \in\left[t_{0}, T\right] .
\end{aligned}
$$

Let $t=T$ on both hand sides of (2.23), we have that

$$
\begin{aligned}
u(T) \leq & \varphi^{-1}\left\{W ^ { - 1 } \left[J ^ { - 1 } \left(J\left(W(a(T))+\int_{\alpha\left(t_{0}\right)}^{\alpha(T)} f_{1}(T, s) d s\right)\right.\right.\right. \\
& +\int_{\alpha\left(t_{0}\right)}^{\alpha(T)} f_{1}(T, s)\left(\int_{t_{0}}^{s} f_{2}(s, \tau) d \tau\right) d s \\
& \left.\left.\left.+\int_{\alpha\left(t_{0}\right)}^{\alpha(T)} f_{1}(T, s)\left(\int_{t_{0}}^{s} f_{2}(s, \tau)\left(\int_{t_{0}}^{\tau} f_{3}(\tau, \xi) d \xi\right) d \tau\right) d s\right)\right]\right\} .
\end{aligned}
$$

Due to the randomness of $T,(2.13)$ is achieved immediately from (2.24). 
Obviously, the most general scenario is that the composite function of unknown function is involved in every layer of iterated integrals. For this kind of integral inequalities, we have the following result.

Theorem 3 Assume that Assumptions 1-5 and the following inequality hold

$$
\begin{aligned}
\varphi(u(t)) \leq & a(t)+\int_{\alpha\left(t_{0}\right)}^{\alpha(t)} f_{1}(t, s) w_{1}(u(s)) d s \\
& +\int_{\alpha\left(t_{0}\right)}^{\alpha(t)} f_{1}(t, s) w_{1}(u(s))\left(\int_{t_{0}}^{s} f_{2}(s, \tau) w_{2}(u(\tau)) d \tau\right) d s \\
& +\int_{\alpha\left(t_{0}\right)}^{\alpha(t)} f_{1}(t, s) w_{1}(u(s))\left(\int_{t_{0}}^{s} f_{2}(s, \tau) w_{2}(u(\tau))\right. \\
& \left.\times\left(\int_{t_{0}}^{\tau} f_{3}(\tau, \xi) w_{3}(u(\xi)) d \xi\right) d \tau\right) d s .
\end{aligned}
$$

Then we have that

$$
u(t) \leq \varphi^{-1}\left\{\Phi_{1}^{-1}\left[\Phi_{2}^{-1}\left(\Phi_{3}^{-1}\left(U_{3}(t)\right)\right)\right]\right\}, \quad \forall t \in\left[t_{0}, T_{3}\right],
$$

where

$$
\begin{aligned}
\Phi_{1}(u):= & \int_{u_{0}}^{u} \frac{d s}{w_{1}\left(\varphi^{-1}(s)\right)}, \quad u>u_{0}, \\
\Phi_{2}(u):= & \int_{u_{0}}^{u} \frac{d s}{w_{2}\left(\varphi^{-1}\left(\Phi_{1}^{-1}(s)\right)\right)}, \quad u>u_{0}, \\
\Phi_{3}(u):= & \int_{u_{0}}^{u} \frac{d s}{w_{3}\left(\varphi^{-1}\left(\Phi_{1}^{-1}\left(\Phi_{2}^{-1}(s)\right)\right)\right)}, \quad u>u_{0}, \\
U_{3}(t):= & \Phi_{3}\left\{\Phi_{2}\left[\Phi_{1}(a(t))+\int_{\alpha\left(t_{0}\right)}^{\alpha(t)} f_{1}(t, s) d s\right]\right. \\
& \left.+\int_{\alpha\left(t_{0}\right)}^{\alpha(t)} f_{1}(t, s)\left(\int_{t_{0}}^{s} f_{2}(s, \tau) d \tau\right) d s\right\} \\
& +\int_{\alpha\left(t_{0}\right)}^{\alpha(t)} f_{1}(t, s)\left(\int_{t_{0}}^{s} f_{2}(s, \tau)\left(\int_{t_{0}}^{\tau} f_{3}(\tau, \xi) d \xi\right) d \tau\right) d s
\end{aligned}
$$

and $\varphi^{-1}, \Phi_{i}^{-1}, i=1,2,3$ are the inverse functions of $\varphi, \Phi_{i}, i=1,2,3$, respectively, and

$$
\begin{aligned}
T_{3}:= & \max \left\{t \in\left[t_{0},+\infty\right) \mid U_{3}(t) \in \operatorname{Dom}\left(\Phi_{3}^{-1}\right),\right. \\
& \left.\Phi_{3}^{-1}\left(U_{3}(t)\right) \in \operatorname{Dom}\left(\Phi_{2}^{-1}\right), \Phi_{2}^{-1}\left(\Phi_{3}^{-1}\left(U_{3}(t)\right)\right) \in \operatorname{Dom}\left(\Phi_{1}^{-1}\right)\right\} .
\end{aligned}
$$

Proof Choose $T \in\left[t_{0}, T_{3}\right]$ arbitrarily. For $\forall t \in\left[t_{0}, T\right]$, we obtain that

$$
\begin{aligned}
\varphi(u(t)) \leq & a(T)+\int_{\alpha\left(t_{0}\right)}^{\alpha(t)} f_{1}(T, s) w_{1}(u(s)) d s \\
& +\int_{\alpha\left(t_{0}\right)}^{\alpha(t)} f_{1}(T, s) w_{1}(u(s))\left(\int_{t_{0}}^{s} f_{2}(s, \tau) w_{2}(u(\tau)) d \tau\right) d s
\end{aligned}
$$




$$
\begin{aligned}
& +\int_{\alpha\left(t_{0}\right)}^{\alpha(t)} f_{1}(T, s) w_{1}(u(s)) \\
& \times\left(\int_{t_{0}}^{s} f_{2}(s, \tau) w_{2}(u(\tau))\left(\int_{t_{0}}^{\tau} f_{3}(\tau, \xi) w_{3}(u(\xi)) d \xi\right) d \tau\right) d s .
\end{aligned}
$$

from (2.25) and the monotonicity of $f_{i}(t, s), i=1,2,3$ on $t$. Let $z_{3}(t)$ be the right-hand side of (2.31), which is a positive and nondecreasing function on $\left[t_{0}, T\right]$ with $z_{3}\left(t_{0}\right)=a(T)$. Then (2.31) is equivalent to

$$
u(t) \leq \varphi^{-1}\left(z_{3}(t)\right), \quad \forall n \in\left[t_{0}, T\right] .
$$

Differentiating $z_{3}$, we can obtain that

$$
\begin{aligned}
\frac{d z_{3}(t)}{d t} \leq & \alpha^{\prime}(t) f_{1}(T, \alpha(t)) w_{1}\left(\varphi^{-1}\left(z_{3}(t)\right)\right)+\alpha^{\prime}(t) f_{1}(T, \alpha(t)) w_{1}\left(\varphi^{-1}\left(z_{3}(t)\right)\right) \\
& \times \int_{t_{0}}^{\alpha(t)} f_{2}(\alpha(t), \tau) w_{2}\left(\varphi^{-1}\left(z_{3}(\tau)\right)\right) d \tau \\
& +\alpha^{\prime}(t) f_{1}(T, \alpha(t)) w_{1}\left(\varphi^{-1}\left(z_{3}(t)\right)\right) \int_{t_{0}}^{\alpha(t)} f_{2}(\alpha(t), \tau) \\
& \times w_{2}\left(\varphi^{-1}\left(z_{3}(\tau)\right)\right)\left(\int_{t_{0}}^{\tau} f_{3}(\tau, \xi) w_{3}\left(\varphi^{-1}\left(z_{3}(\xi)\right)\right) d \xi\right) d \tau,
\end{aligned}
$$

from (2.32) and the monotonicity of $w_{1}, \varphi$ and $z_{3}$, for all $t \in\left[t_{0}, T\right]$. Thus, we have

$$
\begin{aligned}
\frac{d z_{3}(t)}{w_{1}\left(\varphi^{-1}\left(z_{3}(t)\right)\right)} \leq & \left(\alpha^{\prime}(t) f_{1}(T, \alpha(t))+\alpha^{\prime}(t) f_{1}(T, \alpha(t)) \int_{t_{0}}^{\alpha(t)} f_{2}(\alpha(t), \tau)\right. \\
& \times w_{2}\left(\varphi^{-1}\left(z_{3}(\tau)\right)\right) d \tau+\alpha^{\prime}(t) f_{1}(T, \alpha(t)) \int_{t_{0}}^{\alpha(t)} f_{2}(\alpha(t), \tau) \\
& \left.\times w_{2}\left(\varphi^{-1}\left(z_{3}(\tau)\right)\right)\left(\int_{t_{0}}^{\tau} f_{3}(\tau, \xi) w_{3}\left(\varphi^{-1}\left(z_{3}(\xi)\right)\right) d \xi\right) d \tau\right) d t
\end{aligned}
$$

by (2.33) for all $t \in\left[t_{0}, T\right]$. Integrating both sides of the above inequality from $t_{0}$ to $t$, we obtain

$$
\begin{aligned}
\Phi_{1}\left(z_{3}(t)\right) \leq & \Phi_{1}\left(z_{3}\left(t_{0}\right)\right)+\int_{\alpha\left(t_{0}\right)}^{\alpha(t)} f_{1}(T, s) d s \\
& +\int_{\alpha\left(t_{0}\right)}^{\alpha(t)} f_{1}(T, s)\left(\int_{t_{0}}^{s} f_{2}(s, \tau) w_{2}\left(\varphi^{-1}\left(z_{2}(\tau)\right)\right) d \tau\right) d s \\
& +\int_{\alpha\left(t_{0}\right)}^{\alpha(t)} f_{1}(T, s)\left(\int_{t_{0}}^{s} f_{2}(s, \tau) w_{2}\left(\varphi^{-1}\left(z_{2}(\tau)\right)\right)\right. \\
& \left.\times\left(\int_{t_{0}}^{\tau} f_{3}(\tau, \xi) w_{3}\left(\varphi^{-1}\left(z_{2}(\xi)\right)\right) d \xi\right) d \tau\right) d s \\
\leq & \Phi_{1}\left(z_{3}\left(t_{0}\right)\right)+\int_{\alpha\left(t_{0}\right)}^{\alpha(T)} f_{1}(T, s) d s \\
& +\int_{\alpha\left(t_{0}\right)}^{\alpha(t)} f_{1}(T, s)\left(\int_{t_{0}}^{s} f_{2}(s, \tau) w_{2}\left(\varphi^{-1}\left(z_{2}(\tau)\right)\right) d \tau\right) d s
\end{aligned}
$$




$$
\begin{aligned}
& +\int_{\alpha\left(t_{0}\right)}^{\alpha(t)} f_{1}(T, s)\left(\int_{t_{0}}^{s} f_{2}(s, \tau) w_{2}\left(\varphi^{-1}\left(z_{2}(\tau)\right)\right)\right. \\
& \left.\times\left(\int_{t_{0}}^{\tau} f_{3}(\tau, \xi) w_{3}\left(\varphi^{-1}\left(z_{2}(\xi)\right)\right) d \xi\right) d \tau\right) d s,
\end{aligned}
$$

where $\Phi_{1}$ is defined by (2.27). Let $v_{2}(t)$ denote the right-hand side of (2.34), which is a positive and nondecreasing function on $\left[t_{0}, T\right]$ with $v_{2}\left(t_{0}\right)=\Phi_{1}(a(T))+\int_{\alpha\left(t_{0}\right)}^{\alpha(T)} f_{1}(T, s) d s$. Then (2.34) is equivalent to

$$
z_{3}(t) \leq \Phi_{1}^{-1}\left(v_{2}(t)\right), \quad \forall t \in\left[t_{0}, T\right]
$$

Differentiating $v_{2}$, we obtain

$$
\begin{aligned}
\frac{d v_{2}(t)}{d t} \leq & \alpha^{\prime}(t) f_{1}(T, \alpha(t)) \int_{t_{0}}^{\alpha(t)} f_{2}(\alpha(t), \tau) w_{2}\left(\varphi^{-1}\left(\Phi_{1}^{-1}\left(v_{2}(\tau)\right)\right)\right) d \tau \\
& +\alpha^{\prime}(t) f_{1}(T, \alpha(t)) \int_{t_{0}}^{\alpha(t)} f_{2}(\alpha(t), \tau) w_{2}\left(\varphi^{-1}\left(\Phi_{1}^{-1}\left(v_{2}(\tau)\right)\right)\right) \\
& \times\left(\int_{t_{0}}^{\tau} f_{3}(\tau, \xi) w_{3}\left(\varphi^{-1}\left(\Phi_{1}^{-1}\left(v_{2}(\xi)\right)\right)\right) d \xi\right) d \tau, \quad \forall t \in\left[t_{0}, T\right]
\end{aligned}
$$

by (2.35). Applying (2.36) and the monotonicity of $w_{2}, \varphi^{-1}, \Phi_{1}^{-1}$ and $v_{2}$, we can get that

$$
\begin{aligned}
\frac{d v_{2}(t)}{w_{2}\left(\varphi^{-1}\left(\Phi_{1}^{-1}\left(v_{2}(t)\right)\right)\right)} \leq & \left(\alpha^{\prime}(t) f_{1}(T, \alpha(t)) \int_{t_{0}}^{\alpha(t)} f_{2}(\alpha(t), \tau) d \tau\right. \\
& +\alpha^{\prime}(t) f_{1}(T, \alpha(t)) \int_{t_{0}}^{\alpha(t)} f_{2}(\alpha(t), \tau) \\
& \left.\times\left(\int_{t_{0}}^{\tau} f_{3}(\tau, \xi) w_{3}\left(\varphi^{-1}\left(\Phi_{1}^{-1}\left(v_{2}(\xi)\right)\right)\right) d \xi\right) d \tau\right) d t
\end{aligned}
$$

for all $t \in\left[t_{0}, T\right]$. Integrating both sides of the above inequality from $t_{0}$ to $t$, we obtain

$$
\begin{aligned}
\Phi_{2}\left(v_{2}(t)\right) \leq & \Phi_{2}\left(v_{2}\left(t_{0}\right)\right)+\int_{\alpha\left(t_{0}\right)}^{\alpha(t)} f_{1}(T, s)\left(\int_{t_{0}}^{s} f_{2}(s, \tau) d \tau\right) d s \\
& +\int_{\alpha\left(t_{0}\right)}^{\alpha(t)} f_{1}(T, s)\left(\int_{t_{0}}^{s} f_{2}(s, \tau)\left(\int_{t_{0}}^{\tau} f_{3}(\tau, \xi) w_{3}\left(\varphi^{-1}\left(\Phi_{1}^{-1}\left(v_{2}(\xi)\right)\right)\right) d \xi\right) d \tau\right) d s \\
\leq & \Phi_{2}\left(v_{2}\left(t_{0}\right)\right)+\int_{\alpha\left(t_{0}\right)}^{\alpha(T)} f_{1}(T, s)\left(\int_{t_{0}}^{s} f_{2}(s, \tau) d \tau\right) d s+\int_{\alpha\left(t_{0}\right)}^{\alpha(t)} f_{1}(T, s)\left(\int_{t_{0}}^{s} f_{2}(s, \tau)\right. \\
& \left.\times\left(\int_{t_{0}}^{\tau} f_{3}(\tau, \xi) w_{3}\left(\varphi^{-1}\left(\Phi_{1}^{-1}\left(v_{2}(\xi)\right)\right)\right) d \xi\right) d \tau\right) d s
\end{aligned}
$$

for all $t \in\left[t_{0}, T\right]$, where $\Phi_{2}$ is defined by (2.28). Now, let $v_{3}(t)$ be the right-hand side of (2.37), which is a positive and nondecreasing function on $\left[t_{0}, T\right]$ with

$$
\begin{aligned}
v_{3}\left(t_{0}\right) & =\Phi_{2}\left(v_{2}\left(t_{0}\right)\right)+\int_{\alpha\left(t_{0}\right)}^{\alpha(T)} f_{1}(T, s)\left(\int_{t_{0}}^{s} f_{2}(s, \tau) d \tau\right) d s \\
& =\Phi_{2}\left(\Phi_{1}(a(T))+\int_{\alpha\left(t_{0}\right)}^{\alpha(T)} f_{1}(T, s) d s\right)+\int_{\alpha\left(t_{0}\right)}^{\alpha(T)} f_{1}(T, s)\left(\int_{t_{0}}^{s} f_{2}(s, \tau) d \tau\right) d s .
\end{aligned}
$$


Then, (2.37) is equivalent to

$$
v_{2}(t) \leq \Phi_{2}^{-1}\left(v_{3}(t)\right), \quad \forall t \in\left[t_{0}, T\right]
$$

Differentiating $v_{3}$ and applying (2.39), we can obtain that

$$
\begin{aligned}
\frac{d \nu_{3}(t)}{d t} \leq & \alpha^{\prime}(t) f_{1}(T, \alpha(t)) \int_{t_{0}}^{\alpha(t)} f_{2}(\alpha(t), \tau) \\
& \times\left(\int_{t_{0}}^{\tau} f_{3}(\tau, \xi) w_{3}\left(\varphi^{-1}\left(\Phi_{1}^{-1}\left(\Phi_{2}^{-1}\left(v_{3}(\xi)\right)\right)\right)\right) d \xi\right) d \tau
\end{aligned}
$$

for all $t \in\left[t_{0}, T\right]$. By $(2.40)$ and the monotonicity of $w_{3}, \varphi^{-1}, \Phi_{1}^{-1}, \Phi_{2}^{-1}$ and $v_{3}$, we get

$$
\frac{d \nu_{3}(t)}{w_{3}\left(\varphi^{-1}\left(\Phi_{1}^{-1}\left(\Phi_{2}^{-1}\left(v_{3}(t)\right)\right)\right)\right)} \leq\left(\alpha^{\prime}(t) f_{1}(T, \alpha(t)) \int_{t_{0}}^{\alpha(t)} f_{2}(\alpha(t), \tau)\left(\int_{t_{0}}^{\tau} f_{3}(\tau, \xi) d \xi\right) d \tau\right) d t
$$

for all $t \in\left[t_{0}, T\right]$. Integrating both sides of the inequality above, from $t_{0}$ to $t$, we obtain

$$
\Phi_{3}\left(v_{3}(t)\right) \leq \Phi_{3}\left(v_{3}\left(t_{0}\right)\right)+\int_{\alpha\left(t_{0}\right)}^{\alpha(t)} f_{1}(T, s)\left(\int_{t_{0}}^{s} f_{2}(s, \tau)\left(\int_{t_{0}}^{\tau} f_{3}(\tau, \xi) d \xi\right) d \tau\right) d s
$$

for all $t \in\left[t_{0}, T\right]$, where $\Phi_{3}$ is defined by (2.29). By combining (2.32), (2.35), (2.39) and (2.41), we can obtain that

$$
\begin{aligned}
u(t) \leq & \varphi^{-1}\left\{z_{3}(t)\right\} \leq \varphi^{-1}\left\{\Phi_{1}^{-1}\left[v_{2}(t)\right]\right\} \leq \varphi^{-1}\left\{\Phi_{1}^{-1}\left[\Phi_{2}^{-1}\left(v_{3}(t)\right)\right]\right\} \\
\leq & \varphi^{-1}\left\{\Phi_{1}^{-1}\left[\Phi_{2}^{-1}\left(\Phi_{3}^{-1}\left(\Phi_{3}\left(v_{3}\left(t_{0}\right)\right)+\sum_{s=t_{0}}^{n-1} f_{1}(T, s) \int_{t_{0}}^{s} f_{2}(s, \tau) \int_{t_{0}}^{\tau} f_{3}(\tau, \xi)\right)\right)\right]\right\} \\
= & \varphi^{-1}\left\{\Phi _ { 1 } ^ { - 1 } \left[\Phi _ { 2 } ^ { - 1 } \left(\Phi _ { 3 } ^ { - 1 } \left(\Phi _ { 3 } \left(\Phi _ { 2 } \left(\Phi_{1}(a(T))\right.\right.\right.\right.\right.\right. \\
& \left.\left.+\int_{\alpha\left(t_{0}\right)}^{\alpha(T)} f_{1}(T, s) d s\right)+\int_{\alpha\left(t_{0}\right)}^{\alpha(T)} f_{1}(T, s)\left(\int_{t_{0}}^{s} f_{2}(s, \tau) d \tau\right) d s\right) \\
& \left.\left.\left.\left.+\int_{\alpha\left(t_{0}\right)}^{\alpha(t)} f_{1}(T, s)\left(\int_{t_{0}}^{s} f_{2}(s, \tau)\left(\int_{t_{0}}^{\tau} f_{3}(\tau, \xi) d \xi\right) d \tau\right) d s\right)\right)\right]\right\}
\end{aligned}
$$

for all $t \in\left[t_{0}, T\right]$. Let $t=T$ on both hand sides of (2.42), we have

$$
\begin{aligned}
u(T) \leq & \varphi^{-1}\left\{\Phi _ { 1 } ^ { - 1 } \left[\Phi _ { 2 } ^ { - 1 } \left(\Phi _ { 3 } ^ { - 1 } \left(\Phi _ { 3 } \left(\Phi _ { 2 } \left(\Phi_{1}(a(T))\right.\right.\right.\right.\right.\right. \\
& \left.\left.+\int_{\alpha\left(t_{0}\right)}^{\alpha(T)} f_{1}(T, s) d s\right)+\int_{\alpha\left(t_{0}\right)}^{\alpha(T)} f_{1}(T, s)\left(\int_{t_{0}}^{s} f_{2}(s, \tau) d \tau\right) d s\right) \\
& \left.\left.\left.\left.+\int_{\alpha\left(t_{0}\right)}^{\alpha(T)} f_{1}(T, s)\left(\int_{t_{0}}^{s} f_{2}(s, \tau)\left(\int_{t_{0}}^{\tau} f_{3}(\tau, \xi) d \xi\right) d \tau\right) d s\right)\right)\right]\right\} .
\end{aligned}
$$

Since $T$ is chosen arbitrarily in (2.43), thus (2.26) is proved.

As a generalization of Theorem 3, we can obtain the following corollary, which can be proved similarly as Theorem 3 . 
Corollary 1 Assume that Assumptions 1-5 and the following inequality hold

$$
\begin{aligned}
u(t) \leq & a(t)+\int_{\alpha}^{t} f_{1}\left(t, t_{1}\right) w_{1}\left(u\left(t_{1}\right)\right) d t_{1} \\
& +\sum_{i=2}^{n} \int_{\alpha}^{t} f_{1}\left(t, t_{1}\right) w_{1}\left(u\left(t_{1}\right)\right)\left(\int_{\alpha}^{t_{1}} f_{2}\left(t_{1}, t_{2}\right)\right. \\
& \left.\times w_{2}\left(u\left(t_{2}\right)\right)\left(\cdots\left(\int_{\alpha}^{t_{i-1}} f_{i}\left(t_{i-1}, t_{i}\right) w_{i}\left(u\left(t_{i}\right)\right) d t_{i}\right) \cdots\right) d t_{2}\right) d t_{1} .
\end{aligned}
$$

Then we have that

$$
u(t) \leq \varphi^{-1}\left\{\Phi_{1}^{-1}\left[\Phi_{2}^{-1} \cdots\left(\Phi_{n-1}^{-1}\left(\Phi_{n}^{-1}\left(U_{4}(t)\right)\right)\right) \cdots\right]\right\}, \quad \forall t \in\left[t_{0}, T_{4}\right)
$$

where

$$
\begin{aligned}
U_{4}(t):= & \Phi_{n}\left\{\Phi _ { n - 1 } \left[\cdots \Phi_{2}\left(\Phi_{1}(a(t))+\int_{\alpha\left(t_{0}\right)}^{\alpha(t)} f_{1}(t, s)\right)+\cdots\right.\right. \\
& +\int_{\alpha\left(t_{0}\right)}^{\alpha(t)} f_{1}\left(t, t_{1}\right) \\
& \left.\times\left(\int_{t_{0}}^{t_{1}} f_{2}\left(t_{1}, t_{2}\right) \cdots\left(\int_{t_{0}}^{t_{n-3}} f_{n-2}\left(t_{n-3}, t_{n-2}\right) d t_{n-2}\right) d t_{n-3} \cdots d t_{2}\right) d t_{1}\right] \\
& +\int_{\alpha\left(t_{0}\right)}^{\alpha(t)} f_{1}\left(t, t_{1}\right) \\
& \left.\times\left(\int_{t_{0}}^{t_{1}} f_{2}\left(t_{1}, t_{2}\right) \cdots\left(\int_{t_{0}}^{t_{n-2}} f_{n-1}\left(t_{n-2}, t_{n-1}\right) d t_{n-1}\right) d t_{n-2} \cdots d t_{2}\right) d t_{1}\right\} \\
& +\int_{\alpha\left(t_{0}\right)}^{\alpha(t)} f_{1}\left(t, t_{1}\right) \\
& \times\left(\int_{t_{0}}^{t_{1}} f_{2}\left(t_{1}, t_{2}\right) \cdots\left(\int_{t_{0}}^{t_{n-1}} f_{n}\left(t_{n-1}, t_{n}\right) d t_{n}\right) d t_{n-1} \cdots d t_{2}\right) d t_{1},
\end{aligned}
$$

and

$$
\begin{aligned}
& \Phi_{1}(u):=\int_{1}^{u} \frac{d s}{w_{1}\left(\varphi^{-1}(s)\right)}, \quad \Phi_{2}(u):=\int_{1}^{u} \frac{d s}{w_{2}\left(\varphi^{-1}\left(\Phi_{1}^{-1}(s)\right)\right)}, \\
& \Phi_{n-1}(u):=\int_{1}^{u} \frac{d s}{w_{n-1}\left(\varphi^{-1}\left(\Phi_{1}^{-1}\left(\Phi_{2}^{-1}\left(\cdots\left(\Phi_{n-3}^{-1}\left(\Phi_{n-2}^{-1}(s)\right)\right) \cdots\right)\right)\right)\right.}, \\
& \Phi_{n}(u):=\int_{1}^{u} \frac{d s}{w_{n-1}\left(\varphi^{-1}\left(\Phi_{1}^{-1}\left(\Phi_{2}^{-1}\left(\cdots\left(\Phi_{n-2}^{-1}\left(\Phi_{n-1}^{-1}(s)\right)\right) \cdots\right)\right)\right)\right)}
\end{aligned}
$$

and $\varphi^{-1}, \Phi_{i}^{-1}, i=1,2, \ldots, n$ are the inverse functions of $\varphi, \Phi_{i}, i=1,2, \ldots, n$, respectively, and

$$
\begin{aligned}
T_{4}:= & \max \left\{t \in\left[t_{0},+\infty\right) \mid U_{4}\left(T_{4}\right) \in \operatorname{Dom}\left(\Phi_{n}^{-1}\right), \Phi_{n}^{-1}\left(U_{4}\left(T_{4}\right)\right) \in \operatorname{Dom}\left(\Phi_{n-1}^{-1}\right),\right. \\
& \ldots, \Phi_{3}^{-1}\left(\Phi_{4}^{-1}\left(\cdots\left(\Phi_{n-1}^{-1}\left(\Phi_{n}^{-1}\left(U_{4}\left(T_{4}\right)\right)\right)\right) \cdots\right)\right) \in \operatorname{Dom}\left(\Phi_{2}^{-1}\right), \\
& \left.\Phi_{2}^{-1}\left(\Phi_{3}^{-1}\left(\cdots\left(\Phi_{n-1}^{-1}\left(\Phi_{n}^{-1}\left(U_{4}\left(T_{4}\right)\right)\right)\right) \cdots\right)\right) \in \operatorname{Dom}\left(\Phi_{1}^{-1}\right)\right\} .
\end{aligned}
$$




\section{Application}

In this section, we apply our result in Theorem 3 to investigate the robust stability of a class of closed-loop control systems, which demonstrates that our results are handy tools to analyze the qualitative properties of solutions of some nonlinear ordinary differential and integral equations.

For a given control system

$$
\frac{d x(t)}{d t}=A_{0}(t) x(t)+B_{0}(t) u(t)
$$

there is no doubt that controller design plays a pivotal role. Choosing the full state feedback controller $u=-F x$ with the appropriate gain $F$ for (3.1), one can immediately obtain the following stable closed-loop system

$$
\frac{d x(t)}{d t}=A(t) x(t)
$$

where $A(t) \triangleq A_{0}(t)-B_{0}(t) F$. However, in practice, some undesirable system factors, including nonlinear uncertainties and input disturbance, will be involved. As such, before applying the designed controller to real processes, the stability of a closed-loop system against external perturbations must be verified, which is the so-called robust stability analysis.

Consider a perturbed system of (3.2)

$$
\frac{d x(t)}{d t}=A(t) x(t)+f(t, x(\alpha(t)), \sigma(\alpha(t))), \quad t \in\left[t_{0}, \infty\right), x\left(t_{0}\right)=x_{0}
$$

with

$$
\sigma(t)=\theta(t)+\int_{t_{0}}^{t} k(t, s, x(s)) d s
$$

where $\alpha \in C^{1}\left(\left[t_{0}, \infty\right),\left[t_{0}, \infty\right)\right)$ is a nondecreasing function with $\alpha\left(t_{0}\right)=t_{0}, x, y, \theta, \sigma \in$ $C^{1}\left(\mathbf{R}, \mathbf{R}^{r}\right), A(t)$ is a $r \times r$ continuous nonsingular matrix, and the function $f \in C\left(\mathbf{R} \times \mathbf{R}^{r} \times\right.$ $\left.\mathbf{R}^{r}, \mathbf{R}^{r}\right)$ and $k \in C\left(\mathbf{R} \times \mathbf{R} \times \mathbf{R}^{r}, \mathbf{R}^{r}\right)$ satisfy the following conditions

$$
\begin{aligned}
& |f(t, x(\alpha(t)), \sigma(\alpha(t)))| \leq g_{1}(t) e^{-\beta t} w_{1}\left(|x(\alpha(t))| e^{\beta \alpha(t)}\right)(1+|\sigma(\alpha(t))|), \\
& \mid k\left(t, s, x(s) \mid \leq g_{2}(t, s) w_{2}\left(|x(s)| e^{\beta s}\right)\left(1+\int_{t_{0}}^{s} g_{3}(s, \tau) w_{3}\left(|x(\tau)| e^{\beta \tau}\right) d \tau\right),\right.
\end{aligned}
$$

where $\beta>0$ is a constant, $g_{1} \in C\left(\left[t_{0}, \infty\right), \mathbf{R}_{+}\right)$and $g_{i} \in C\left(\left[t_{0}, \infty\right) \times\left[t_{0}, \infty\right), \mathbf{R}_{+}\right), i=2,3$, are nondecreasing in $t$ for fixed $s \in\left[t_{0}, \infty\right), w_{i}(u), i=1,2,3$, are positive and continuous functions defined on $[0, \infty)$. In general, the perturbation term $f(t, x(\alpha(t)), \sigma(\alpha(t)))$ could result from modeling errors, aging, uncertainties, disturbances, or some other reasons. Suppose that the nominal system (3.2) has a uniformly asymptotically stable equilibrium at the origin, we next exploit the stability of the perturbed system (3.3). The result is presented in the following proposition. 
Proposition 1 If there exists a constant $C>0$ such that the fundamental solution matrix $X(t)$ of the linear system (3.2) satisfies

$$
\left|X(t) X^{-1}(s)\right| \leq C \exp (-\beta(t-s)), \quad 0 \leq s \leq t \leq \infty,
$$

then we have that

$$
\left|x_{\sigma}\left(t, t_{0}, x_{0}\right)\right| \leq \exp (-\beta t)\left\{\Phi_{4}^{-1}\left[\Phi_{5}^{-1}\left(\Phi_{6}^{-1}\left(U_{4}(t)\right)\right)\right]\right\}, \quad \forall t \in\left[t_{0},+\infty\right)
$$

where $x_{\sigma}\left(t, t_{0}, x_{0}\right)$ is a solution of the control system (3.3) with (3.4) and

$$
\begin{aligned}
f_{1}(s):= & \frac{C g_{1}\left(\alpha^{-1}(s)\right) e^{\beta}(1+|\theta(s)|)}{\alpha^{\prime}\left(\alpha^{-1}(s)\right)} \\
\Phi_{4}(u): & \int_{1}^{u} \frac{d s}{w_{1}(s)}, \quad u>0 \\
\Phi_{5}(u): & \int_{1}^{u} \frac{d s}{w_{2}\left(\Phi_{1}^{-1}(s)\right)}, \quad u>0 \\
\Phi_{6}(u): & \int_{1}^{u} \frac{d s}{w_{3}\left(\Phi_{1}^{-1}\left(\Phi_{2}^{-1}(s)\right)\right)}, \quad u>0, \\
U_{4}(t):= & \Phi_{6}\left\{\Phi_{5}\left[\Phi_{4}\left(\left|x_{0}\right| C \exp \left(\beta t_{0}\right)\right)+\int_{t_{0}}^{\alpha(t)} f_{1}(s) d s\right]+\int_{t_{0}}^{\alpha(t)} f_{1}(s)\left(\int_{t_{0}}^{s} g_{2}(s, \tau) d \tau\right) d s\right\} \\
& +\int_{t_{0}}^{\alpha(t)} f_{1}(s)\left[\int_{t_{0}}^{s} g_{2}(s, \tau)\left(\int_{t_{0}}^{\tau} g_{3}(\tau, \xi) d \xi\right) d \tau\right] d s
\end{aligned}
$$

and $\Phi_{i}^{-1}, i=4,5,6$ are the inverse functions of $\Phi_{i}, i=4,5,6$, respectively, and

$$
\begin{aligned}
T_{4}:= & \max \left\{t \in\left[t_{0},+\infty\right) \mid U_{4}(t) \in \operatorname{Dom}\left(\Phi_{6}^{-1}\right),\right. \\
& \left.\Phi_{6}^{-1}\left(U_{4}(t)\right) \in \operatorname{Dom}\left(\Phi_{5}^{-1}\right), \Phi_{5}^{-1}\left(\Phi_{6}^{-1}\left(U_{4}(t)\right)\right) \in \operatorname{Dom}\left(\Phi_{4}^{-1}\right)\right\} .
\end{aligned}
$$

Further, if there exists a positive constant $b$ such that

$$
\left\{\Phi_{4}^{-1}\left[\Phi_{5}^{-1}\left(\Phi_{6}^{-1}\left(U_{4}(t)\right)\right)\right]\right\} \leq b,
$$

any solution of the control system (3.3) with (3.4) is exponentially asymptotically stable.

Proof Firstly, we can obtain the solution of (3.3) with (3.4)

$$
\begin{aligned}
x_{\sigma}\left(t, t_{0}, x_{0}\right)= & X(t) X^{-1}\left(t_{0}\right) x_{0} \\
& +\int_{t_{0}}^{t} X(s) X^{-1}(s+1) f\left(s, x_{\sigma}\left(\alpha(s), t_{0}, x_{0}\right), \sigma(\alpha(s))\right) d s,
\end{aligned}
$$

by using the variation of constants formula. Then we have that

$$
\begin{aligned}
\left|x_{\sigma}\left(t, t_{0}, x_{0}\right)\right| \leq & \left|x_{0}\right| C \exp \left(-\beta\left(t-t_{0}\right)\right)+\int_{t_{0}}^{t} C \exp (-\beta(t-s-1)) \\
& \times g_{1}(s) e^{-\beta s} w_{1}\left(\left|x_{\sigma}\left(\alpha(s), t_{0}, x_{0}\right)\right| e^{\beta s}\right)(1+|\sigma(\alpha(s))|),
\end{aligned}
$$


by conditions (3.5) and (3.7) from (3.14). Further, by using conditions (3.6), we can obtain that

$$
\begin{aligned}
& \left|x_{\sigma}\left(t, t_{0}, x_{0}\right)\right| \\
& \leq\left|x_{0}\right| C \exp \left(-\beta\left(t-t_{0}\right)\right)+\int_{t_{0}}^{t} C \exp (-\beta(t-1)) g_{1}(s) \\
& \quad \times w_{1}\left(\left|x_{\sigma}\left(\alpha(s), t_{0}, x_{0}\right)\right| e^{\beta \alpha(s)}\right)\left\{1+|\theta(\alpha(s))|+\int_{t_{0}}^{\alpha(s)} g_{2}(\alpha(s), \tau)\right. \\
& \left.\quad \times w_{2}\left(\left|x_{\sigma}\left(\tau, t_{0}, x_{0}\right)\right| e^{\beta \tau}\right)\left(1+\int_{t_{0}}^{\tau} g_{3}(\tau, \xi) w_{3}\left(\left|x_{\sigma}\left(\xi, t_{0}, x_{0}\right)\right| e^{\beta \xi}\right) d \xi\right) d \tau\right\} d s
\end{aligned}
$$

from (3.4) and (3.15). Then we have that

$$
\begin{aligned}
& \left|x_{\sigma}\left(t, t_{0}, x_{0}\right)\right| \\
& \leq\left|x_{0}\right| C \exp \left(-\beta\left(t-t_{0}\right)\right)+e^{-\beta t} \int_{t_{0}}^{\alpha(t)} C e^{\beta} g_{1}\left(\alpha^{-1}(\eta)\right) \\
& \quad \times w_{1}\left(\left|x_{\sigma}\left(\eta, t_{0}, x_{0}\right)\right| e^{\beta \eta}\right) \times\left\{1+|\theta(\eta)|+\int_{t_{0}}^{\eta} g_{2}(\eta, \tau) w_{2}\left(\left|x_{\sigma}\left(\tau, t_{0}, x_{0}\right)\right| e^{\beta \tau}\right)\right. \\
& \left.\quad \times\left(1+\int_{t_{0}}^{\tau} g_{3}(\tau, \xi) w_{3}\left(\left|x_{\sigma}\left(\xi, t_{0}, x_{0}\right)\right| e^{\beta \xi}\right) d \xi\right) d \tau\right\} \frac{d \eta}{\alpha^{\prime}\left(\alpha^{-1}(\eta)\right)},
\end{aligned}
$$

where we use the change $\eta=\alpha(s)$. Let $u(t)=\left|x_{\sigma}\left(n, t_{0}, x_{0}\right)\right| \exp (\beta t),(3.16)$ can be rewritten as

$$
\begin{aligned}
u(t) \leq & \left|x_{0}\right| C \exp \left(\beta t_{0}\right)+\int_{t_{0}}^{\alpha(t)} C e^{\beta} g_{1}\left(\alpha^{-1}(s)\right) w_{1}(u(s))\{1+|\theta(s)| \\
& \left.+\int_{t_{0}}^{s} g_{2}(s, \tau) w_{2}(u(\tau))\left(1+\int_{t_{0}}^{\tau} g_{3}(\tau, \xi) w_{3}(u(\xi)) d \xi\right) d \tau\right\} \frac{d s}{\alpha^{\prime}\left(\alpha^{-1}(s)\right)} .
\end{aligned}
$$

Letting $a(t)=\left|x_{0}\right| C \exp \left(\beta t_{0}\right)$, we have

$$
\begin{aligned}
u(t) \leq & a(t)+\int_{t_{0}}^{\alpha(t)} f_{1}(s) w_{1}(u(s)) d s+\int_{t_{0}}^{\alpha(t)} f_{1}(s) w_{1}(u(s))\left(\int_{t_{0}}^{s} f_{2}(s, \tau) w_{2}(u(\tau)) d \tau\right) d s \\
& +\int_{t_{0}}^{\alpha(t)} f_{1}(s) w_{1}(u(s)) \\
& \times\left[\int_{t_{0}}^{s} f_{2}(s, \tau) w_{2}(u(\tau))\left(\int_{t_{0}}^{\tau} f_{3}(\tau, \xi) w_{3}(u(\xi)) d \xi\right) d \tau\right] d s,
\end{aligned}
$$

from (3.10) and (3.17). Applying the result of Theorem 3, the inequality

$$
\left|x_{\sigma}\left(t, t_{0}, x_{0}\right)\right| \leq \exp (-\beta t)\left\{\Phi_{4}^{-1}\left[\Phi_{5}^{-1}\left(\Phi_{6}^{-1}\left(U_{4}(t)\right)\right)\right]\right\}
$$

is proved.

If there exists a positive constant $b$ such that

$$
\left\{\Phi_{4}^{-1}\left[\Phi_{5}^{-1}\left(\Phi_{6}^{-1}\left(U_{4}(t)\right)\right)\right]\right\} \leq b, \quad \forall n \in \mathbf{N}
$$


then we have that

$$
\left|x_{\sigma}\left(t, t_{0}, x_{0}\right)\right| \leq b \exp (-\beta t)
$$

i.e., the nonlinear control system (3.3) with (3.4) is exponentially asymptotically stable.

\section{Competing interests}

The authors declare that they have no competing interests.

\section{Authors' contributions}

All the authors have contributed in all the paper parts.

\section{Author details}

'Department of Mathematics, Hechi University, Yizhou, Guangxi 546300, P.R. China. ${ }^{2}$ Department of Electrical and

Computer Engineering, National University of Singapore, 4 Engineering drive 3, Singapore, 117583, Singapore.

\section{Acknowledgements}

This research was supported by the National Natural Science Foundation of China (Project No. 11161018), the SERC Research Grant (Project No. 092101 00558), the Guangxi Natural Science Foundation (Project No. 0991265 and 2012GXNSFAA053009), the Scientific Research Foundation of the Education Department of Guangxi Province of China (Project No. 201106LX599), and the Key Discipline of Applied Mathematics of Hechi University of China (200725).

Received: 18 September 2012 Accepted: 25 July 2013 Published: 9 August 2013

\section{References}

1. Gronwall, TH: Note on the derivatives with respect to a parameter of the solutions of a system of differential equations. Ann. Math. 20, 292-296 (1919)

2. Bellman, R: The stability of solutions of linear differential equations. Duke Math. J. 10, 643-647 (1943)

3. Bihari, IA: A generalization of a lemma of Bellman and its application to uniqueness problem of differential equation. Acta Math. Acad. Sci. Hung. 7, 81-94 (1956)

4. Pachpatte, BG: Inequalities for Differential and Integral Equations. Academic Press, London (1998)

5. Lipovan, O: A retarded Gronwall-like inequality and its applications. J. Math. Anal. Appl. 252, 389-401 (2000)

6. Dragomir, SS, Kim, YH: On certain new integral inequalities and their applications. J. Inequal. Pure Appl. Math. 3(4), Article ID 65 (2002)

7. Bainov, D, Simeonov, P: Integral Inequalities and Applications. Kluwer Academic, Dordrecht (1992)

8. Kim, Bl: On some Gronwall type inequalities for a system integral equation. Bull. Korean Math. Soc. 42(4), 789-805 (2005)

9. Cho, YJ, Dragomir, SS, Kim, YH: On some integral inequalities with iterated integrals. J. Korean Math. Soc. 43(3), 563-578 (2006)

10. Agarwal, RP, Ryoo, CS, Kim, YH: New integral inequalities for iterated integrals with applications. J. Inequal. Appl. 2007, Article ID 24385 (2007)

11. Abdeldaim, A, Yakout, M: On some new integral inequalities of Gronwall-Bellman-Pachpatte type. Appl. Math. Comput. 217, 7887-7899 (2011)

12. Agarwal, RP, Deng, S, Zhang, W: Generalization of a retarded Gronwall-like inequality and its applications. Appl. Math. Comput. 165, 599-612 (2005)

13. Agarwal, RP, Kim, YH, Sen, SK: New retarded integral inequalities with applications. J. Inequal. Appl. 2008, Article ID 908784 (2008)

14. Cheung, WS: Some new nonlinear inequalities and applications to boundary value problems. Nonlinear Anal. 64, 2112-2128 (2006)

15. Zhao, X, Meng, F: On some advanced integral inequalities and their applications. JIPAM. J. Inequal. Pure Appl. Math. 6(3), Article ID 60 (2005)

16. Cheung, WS, Ma, QH: On certain new Gronwall-Ou-lang type integral inequalities in two variables and their applications. J. Inequal. Appl. 2005(4), 347-361 (2005)

17. Kim, YH: On some new integral inequalities for functions in one and two variables. Acta Math. Sin. 21, 423-434 (2005)

18. Pachpatte, BG: ON certain nonlinear integral inequalities involving iterated integrals. Tamkang J. Math. 37(3), 261-271 (2006)

19. Wang, WS: A generalized retarded Gronwall-like inequality in two variables and applications to BVP. Appl. Math. Comput. 191, 144-154 (2007)

20. $\mathrm{Ma}, \mathrm{QH}$, Pečarić, J: On certain new nonlinear retarded integral inequalities for functions in two variables and their applications. J. Korean Math. Soc. 45(1), 121-136 (2008)

21. Wang, WS, Shen, C: On a generalized retarded integral inequality with two variables. J. Inequal. Appl. 2008, Article ID 518646 (2008)

22. Wang, WS, Li, Z, Li, Y, Huang, Y: Nonlinear retarded integral inequalities with two variables and applications. J. Inequal. Appl. 2010, Article ID 240790 (2010)

23. Wang, WS, Luo, RC, Li, Z: A new nonlinear retarded integral inequality and its application. J. Inequal. Appl. 2010, Article ID 462163 (2010) 
doi:10.1186/1029-242X-2013-376

Cite this article as: Wang et al.: Generalized retarded nonlinear integral inequalities involving iterated integrals and an application. Journal of Inequalities and Applications 2013 2013:376.

Submit your manuscript to a SpringerOpen ${ }^{\circ}$ journal and benefit from:

- Convenient online submission

- Rigorous peer review

- Immediate publication on acceptance

- Open access: articles freely available online

- High visibility within the field

- Retaining the copyright to your article

Submit your next manuscript at $\gg$ springeropen.com 